Boise State University

ScholarWorks

6-4-2015

\title{
Confocal Raman Spectroscopy and AFM for Evaluation of Sidewalls in Type II Superlattice FPAs
}

D. A. Tenne

Boise State University

\section{Publication Information}

Tenne, D. A. (2015). "Confocal Raman Spectroscopy and AFM for Evaluation of Sidewalls in Type II Superlattice FPAs". Proceedings of SPIE, 9451, 94510R-1 - 94510R-12. http://dx.doi.org/10.1117/12.2178162

Copyright 2015 Society of Photo-Optical Instrumentation Engineers. One print or electronic copy may be made for personal use only. Systematic reproduction and distribution, duplication of any material in this paper for a fee or for commercial purposes, or modification of the content of the paper are prohibited. doi: $10.1117 / 12.2178162$ 


\title{
Confocal Raman Spectroscopy and AFM for Evaluation of Sidewalls in Type II Superlattice FPAs
}

\author{
T.J. Rotter *'T. Busani, P. Rathi, F. Jaeckel, P. A. Reyes, K. J. Malloy and A. A. Ukhanov \\ Actoprobe LLC, 801 University SE, Suite 100, Albuquerque, NM 87106 \\ E. Plis and S. Krishna \\ Skinfrared LLC, Lobo Venture Lab 801, University Blvd., Suite 101, Albuquerque NM 87106 \\ M. Jaime-Vasquez, N. F. Baril, and J. D. Benson
}

US Army RDECOM CERDEC Night Vision and-Electronic Sensors Directorate, RDER NVS-IF 10221 Burbeck Road, Fort Belvoir, VA 22060-5806

D. A. Tenne

Boise State University, Department of Physics, 1910 University Dr., Boise, ID 83725

\begin{abstract}
We propose to utilize confocal Raman spectroscopy combined with high resolution atomic force microscopy (AFM) for nondestructive characterisation of the sidewalls of etched and passivated small pixel $(24 \mu \mathrm{m} \times 24 \mu \mathrm{m})$ focal plane arrays (FPA) fabricated using LW/LWIR InAs/GaSb type-II strained layer superlattice (T2SL) detector material. Special high aspect ratio Si and GaAs AFM probes, with tip length of $13 \mu \mathrm{m}$ and tip aperture less than $7^{\circ}$, allow characterisation of the sidewall morphology. Confocal microscopy enables imaging of the sidewall profile through optical sectioning. Raman spectra measured on etched T2SL FPA single pixels enable us to quantify the non-uniformity of the mesa delineation process.
\end{abstract}

Keywords: Infrared Detection, AFM, Confocal Raman spectroscopy, InAs/GaSb Superlattice, Focal Plane Array, Dual-band imaging

\section{INTRODUCTION}

Multicolor detector capabilities in the infrared are highly beneficial for various military and civil applications involving identification of temperature differences and determination of the thermal characteristics of an object. Among these applications are homeland security, industrial imaging, banks, facility security, custom control and law enforcement. Detectors based on interband (Mercury-Cadmium-Telluride, MCT) and intersubband (quantum well infrared detectors, QWIPs) transitions have been the dominant technologies for such applications, ${ }^{18} .{ }^{30}$ Difficulties in the epitaxial growth of MCT and low electron effective mass $\left(\sim 0.009 \mathrm{~m}_{\circ}\right)$ resulting in large dark current due to tunneling especially at longer wavelengths ${ }^{26}$ affect the development of multispectral cameras based on MCT. Compared to MCT detectors, QWIPs have a number of advantages, including the use of standard manufacturing techniques based on mature GaAs growth and processing technologies, highly uniform and well controlled MBE growth on larger area GaAs wafers (6"), high yield and thus low cost, more thermal stability. However, they have larger dark currents and lower quantum efficiency compared to the interband devices.

On the contrary, the basic material properties of InAs/GaSb type-II strained layer superlattices (T2SL) provide a prospective benefit in the realization of dual-color imagers. The strain in the InAs/GaSb T2SL system facilitates suppression of interband tunneling ${ }^{29}$ and Auger recombination. ${ }^{8}$ The larger effective mass $\left(\sim 0.04 \mathrm{~m}_{\circ}\right)$ in T2SL leads to a reduction of tunneling currents compared with MCT detectors of the same bandgap. ${ }^{29}$ By optimizing the oscillator strength in this material system, a large quantum efficiency and responsivity can be

Presenting author

Infrared Technology and Applications XLI, edited by Bjørn F. Andresen,

Gabor F. Fulop, Charles M. Hanson, Paul R. Norton, Proc. of SPIE Vol. 9451

94510R - (c) 2015 SPIE · CCC code: 0277-786X/15/\$18 - doi: 10.1117/12.2178162

Proc. of SPIE Vol. $945194510 R-1$ 
obtained. Moreover, the commercial availability of substrates with good electro-optical homogeneity, and without large cluster defects, also offers advantages for T2SL technology.

High performance InAs/GaSb T2SL detectors have been reported for the mid-wave infrared (MWIR) ${ }^{22,23,38}$ long-wave IR (LWIR), ${ }^{5,7,32}$ and very long wave IR (VLWIR) ${ }^{37}$ spectral regions. Moreover, mega-pixel FPAs, i.e. FPAs of sizes up to $1024 \times 1024$ have been demonstrated. ${ }^{9,10}$ Multiband T2SL structures were also realized, including short-wave IR(SW)/MWIR, ${ }^{11} \mathrm{MW} / \mathrm{MWIR},{ }^{24} \mathrm{MW} / \mathrm{LWIR},{ }^{13,17,21} \mathrm{LW} / \mathrm{LWIR},{ }^{14,15}$ and SW/MW/LWIR ${ }^{6}$ detectors and focal plane arrays (FPAs).

However, the performance parameters of T2SL detectors, in particular, the dark current densities, are still lower compared with the MCT benchmark (em Rule 07). ${ }^{25}$ To realize T2SL-based focal plane arrays (FPAs) with high resolution the number of FPA pixels needs to increase with simultaneous scaling of the lateral dimensions of individual pixels. Then the FPA performance, with typical mesa dimensions of individual FPA pixels of $20 \mu \mathrm{m} \times 20 \mu \mathrm{m}$, is strongly dependent on surface effects due to a large pixel surface/volume ratio. Thus, the development of the mesa delineation process followed by the appropriate passivation is crucial for the realization of high performance T2SL multicolor detectors.

To characterize the quality of etched sidewalls and conformal uniformity of the passivation layer, several techniques may be utilized, such as imaging by transmission electron microscopy (TEM) or scanning electron microscopy (SEM). While producing reliable results, both of these techniques require sample preparation, often destructive, to access the vertical etched or passivated surfaces of the detector. Thus, the device fabrication process is significantly slowed due to the impeded feedback on sidewall morphology after etch and passivation procedures.

Several variations of the AFM technique have been developed for vertical sidewall profiling of micro- and nanostructures. ${ }^{16,19,36}$ These AFM-based methods can probe the surface defects on close to vertical surfaces with good accuracy, however, they are unable to characterize the chemical composition of the defects. In this paper we present a technique based on confocal Raman spectroscopy combined with high resolution AFM that enables non destructive characterization of sidewall profiles as well as chemical composition of etched and passivated small $(24 \mu \mathrm{m} \times 24 \mu \mathrm{m})$ focal plane array (FPA) features fabricated from LW/LWIR InAs/GaSb type-II strained layer superlattice (T2SL) detector material. Furthermore, the combination of Atomic Force Microscope with Tip-Enhanced Raman Spectroscopy (AFM/TERS) is discussed. These techniques will be of great advantage for efficient T2SL FPA development.

\section{EXPERIMENTAL}

\subsection{Confocal Raman AFM Instrument}

The experimental set up, the confocal Raman AFM microscope, is presented in Figure 1. The AFM utilizes special high aspect ratio probes of long length (up to $13 \mu \mathrm{m}$, manufactured by Actoprobe) The confocal Raman microscope (ACTO-S, developed by Actoprobe LLC) can be equipped with various long-range objectives, from $10 \times$ to $100 \times$, with numerical apertures 0.4-0.9. It utilizes a Horiba Jobin Yvon T64000 triple spectrometer equipped with a liquid nitrogen cooled multichannel CCD detector. The $441.6 \mathrm{~nm}$ line of a He-Cd laser was used for Raman excitation. Measurements were performed in backscattering geometry at room temperature. The He-Cd laser excitation is applied at $45^{\circ}$ with respect to the sample in order to reduce elastically scattered laser light collected by the objective.

The T2SL FPA was mounted on the AFM X-Y-Z stage and scanned in raster-scan fashion. Using the confocal microscope with a $405 \mathrm{~nm}$ blue laser to illuminate the sample we scanned a FPA with $6.7 \mu \mathrm{m}$ deep trenches, see Figure 2, from top of the pixel to bottom of the etched trench in $250 \mathrm{~nm}$ slices using $18 \mu \mathrm{m} \times 18 \mu \mathrm{m}$ scan range. Initially we scanned a slice on the top of the pixel, then moved the objective (focal point) by $250 \mathrm{~nm}$ down, using the $\mathrm{Z}$ piezo, and scanned the second slice. We scanned altogether 33 slices (optical sectioning). The microscope was equipped with a $100 \times$ objective with $0.9 \mathrm{NA}$, and the confocal aperture was provided by a single mode fiber of $3 \mu \mathrm{m}$ mode field diameter (i.e. the confocal aperture size was $3 \mu \mathrm{m}$ ). The data was collected through an ultra sensitive femto-watt silicon detector. Figure 2 shows a FPA side wall profile extracted from line cross sections of different slices. The confocal imaging technique provides high resolution optical images (even with spectral information if a spectrometer is used) which can access features at places where the AFM tip cannot reach.

Proc. of SPIE Vol. $945194510 R-2$ 


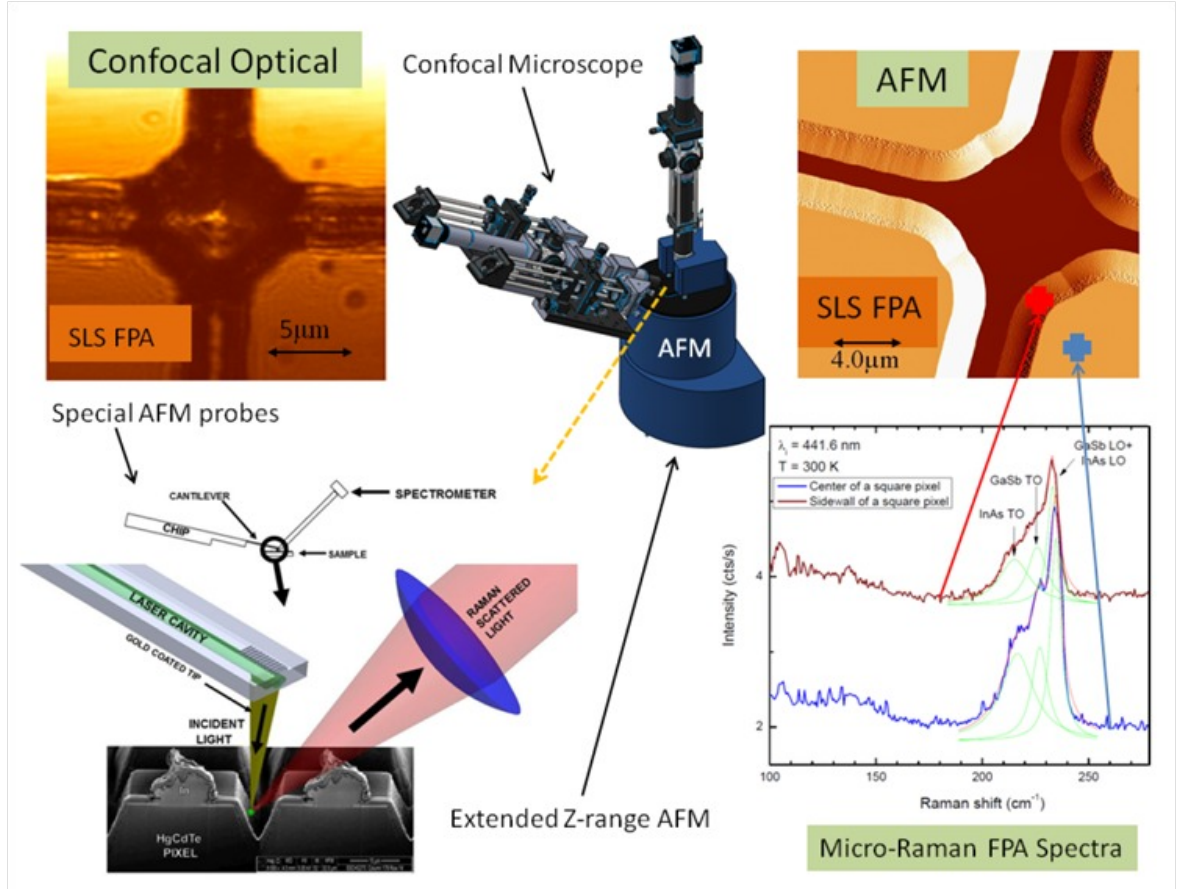

Figure 1. Overview of Raman AFM instrument for T2SL FPA characterization.

\subsection{Probe fabrication}

AFM sidewall characterization of MWIR and LWIR T2SL FPAs is difficult with conventional AFM probes. Their low aspect ratio does not allow them to reach into the deep trenches of the FPAs. The Actoprobe team developed its own High Aspect Ratio AFM probes to accomplish this challenging task.

Standard (low aspect ratio) Si probes were mounted in the sample holder of a Zeiss Auriga vacuum chamber equipped with a Gemini Column for e-beam imaging and a Ga gun for ion-milling. Ion currents of 50-500 pA were applied for milling, and the current and milling time were changed according to the dose. A lower current was used to sharpen the probe tip. We also fabricated Pt coated probes for conductivity measurements. The $\mathrm{Pt}$ was supplied as Pt organometallic gas through a nozzle. The nozzle allows a controlled deposition of $\mathrm{Pt}$ on the probe tip either by the e-beam or by the ion-beam. The Pt coating also reinforces the contact between the tip and the cantilever, which might be beneficial for very thin cantilevers. Specific characteristics of the high aspect ratio probes such as length, tip aperture and tip radius depend to some extend on the low aspect ratio AFM probes from which they were made. Here we present high aspect ratio probes fabricated from different commercially available probes: PPP-NCRL Nanosensors ${ }^{\mathrm{TM}}$, and TR-400 Olympus. SEM images of high-aspect ratio probes fabricated by Actoprobe are shown in Figures 3 and 4.

\subsection{Material growth and fabrication}

The detector material, on which the AFM/TERS measurements were performed, was grown on a n-type (Tedoped) GaSb (001) epi-ready substrate using elemental source molecular beam epitaxy (MBE) in a Veeco GEN 10 system equipped with valved cracker sources for group V ( $\mathrm{Sb}_{2}$ and $\left.\mathrm{As}_{2}\right)$ and $\mathrm{Ga} / \mathrm{In} \mathrm{SUMO} 囚$ cells. The device structure is composed of two $4 \mu \mathrm{m}$ thick T2SL absorbers with the same cut-off wavelength $(\sim 9 \mu \mathrm{m}$ at $68 \mathrm{~K})$ separated by a $100 \mathrm{~nm}$ barrier layer. The LWIR absorbers as well as the barrier layer were p-type doped with carrier concentrations of $1 \times 10^{16} \mathrm{~cm}^{-3}$. The p-type $\left(2 \times 10^{18} \mathrm{~cm}^{-3}\right)$ bottom contact layer with the same T2SL composition and thickness as the LWIR absorbers was grown on a $200 \mathrm{~nm}$ thick p-type $\left(2 \times 10^{18} \mathrm{~cm}^{-3}\right) \mathrm{GaSb}$ buffer layer. The top contact layer (150 nm thick), with the same T2SL composition and doping level as the bottom contact layer, capped the structure. 


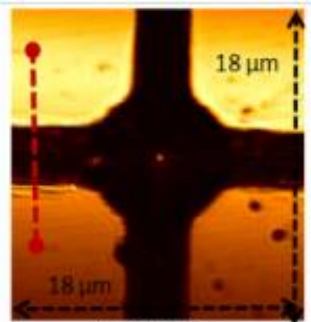

At pixel top

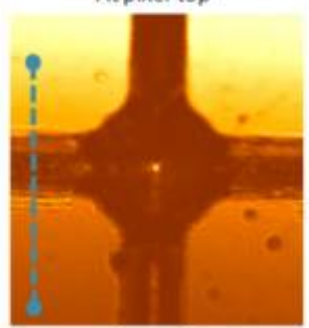

At 1 um from pixel top

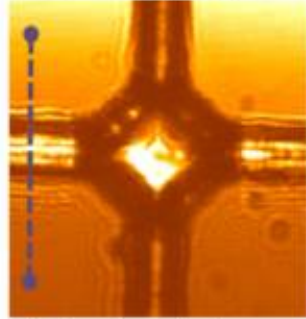

At $4 \mu \mathrm{m}$ from pixel top

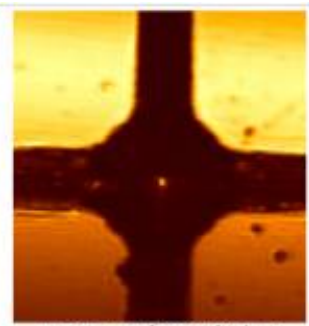

At $250 \mathrm{~nm}$ from pixel top

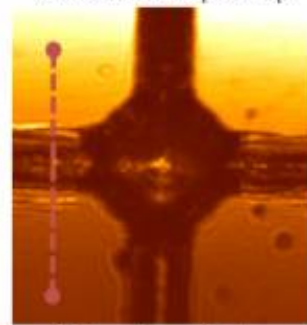

At $2 \mu \mathrm{m}$ from pixel top

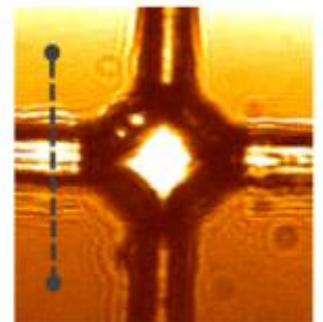

At $5 \mu \mathrm{m}$ from pixel top

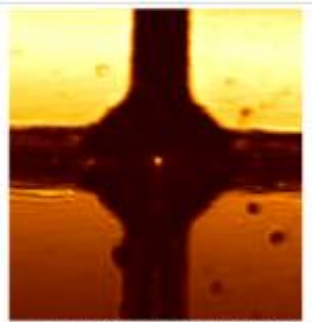

At $500 \mathrm{~nm}$ from pixel top

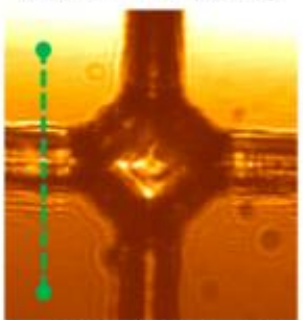

At 3 um from pixel top

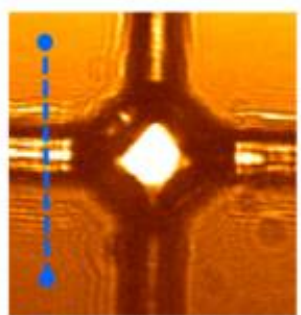

At $6 \mu \mathrm{m}$ from pixel top

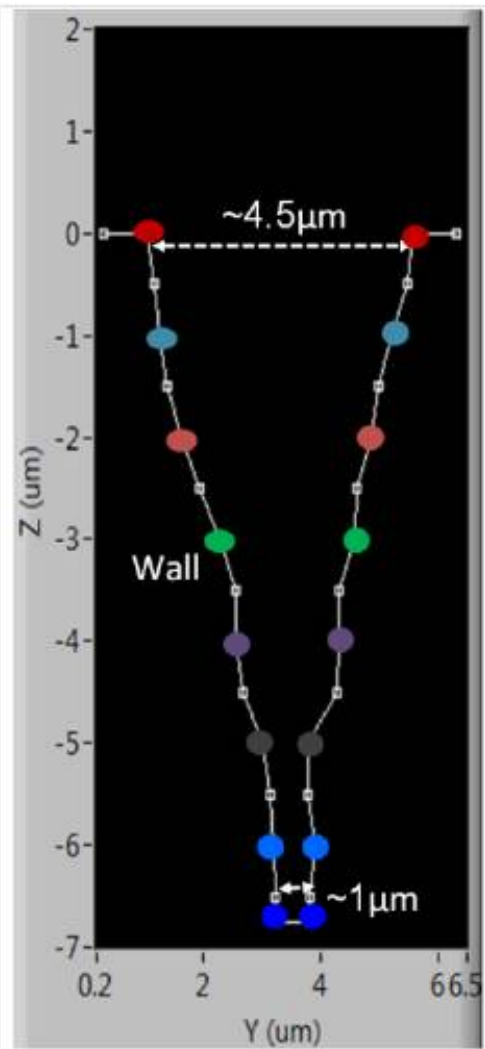

Figure 2. Confocal images of FPA with $6.7 \mu \mathrm{m}$ deep trenches (left). The image size is $18 \mu \mathrm{m} \times 18 \mu \mathrm{m}$. The sample is scanned at different depths starting from the pixel top. The dashed lines (different colors) inside the figures correspond to the cross sections used to create the FPA side wall profile (right). Each data point in the profile corresponds to one of the dashed lines in the confocal image. This is illustrated by the different colors. The data suggests the resolution to be better than $50 \mathrm{~nm}$ lateral and $150 \mathrm{~nm}$ vertical.
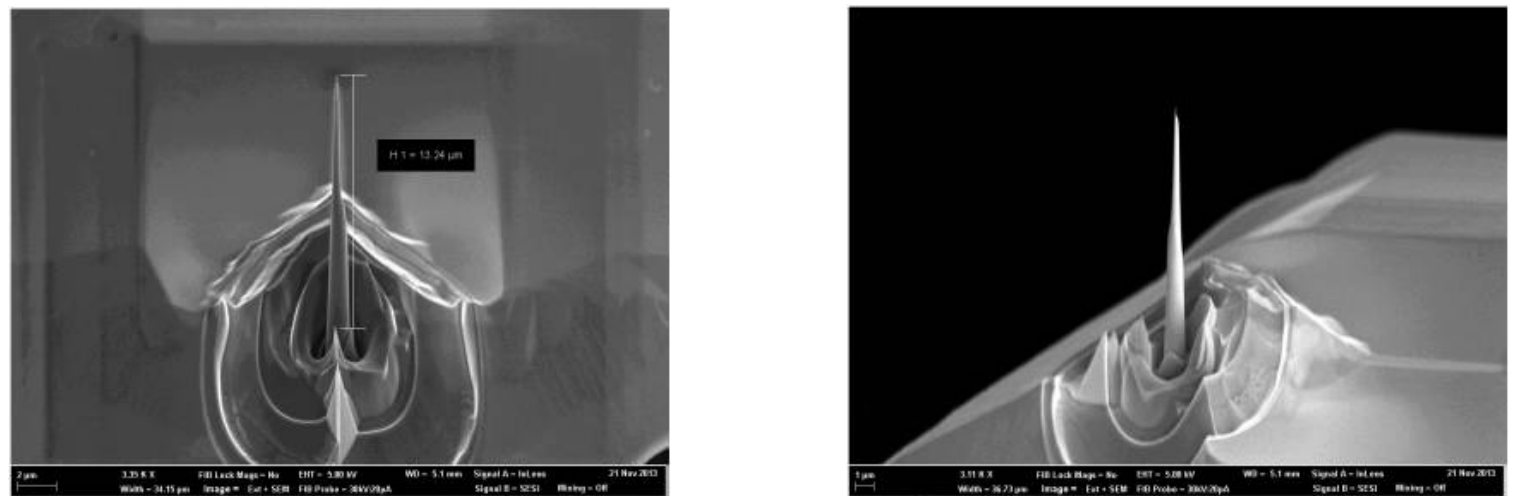

Figure 3. SEM image (left) and enlarged SEM image (right) of high-aspect-ratio probe tips fabricated from PPP-NCRL Nanosensors $^{\mathrm{TM}}$ probes. 

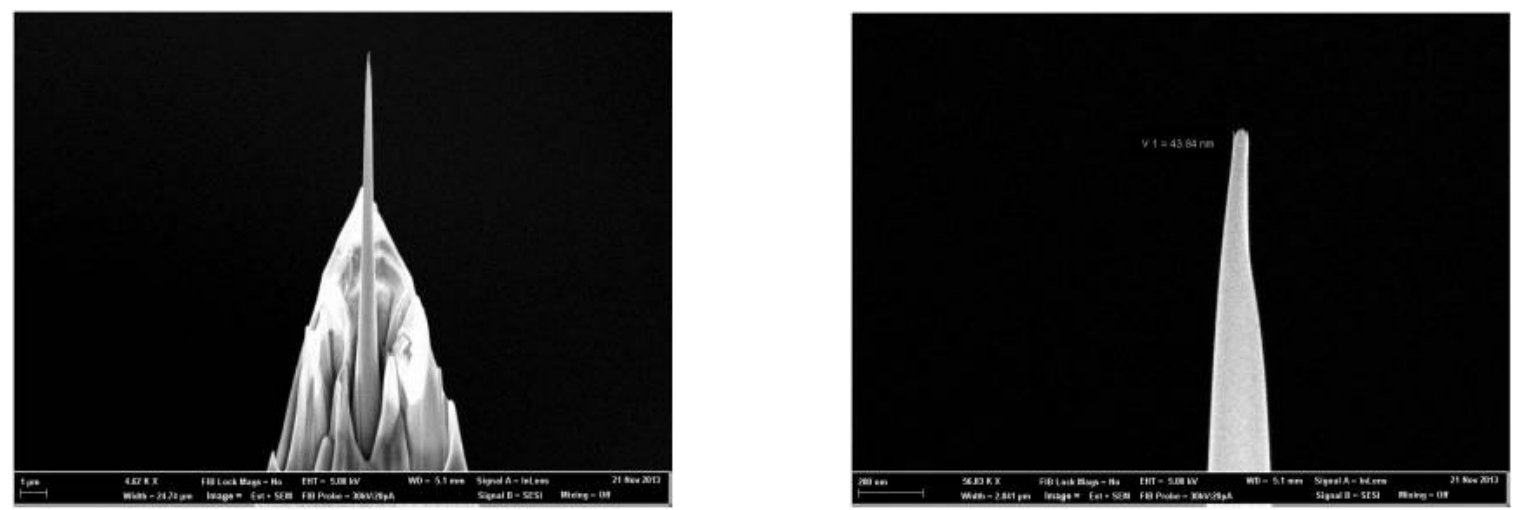

Figure 4. SEM image (left) and enlarged SEM image (right) of high-aspect-ratio probe tips fabricated from TR-400 Olympus probes.
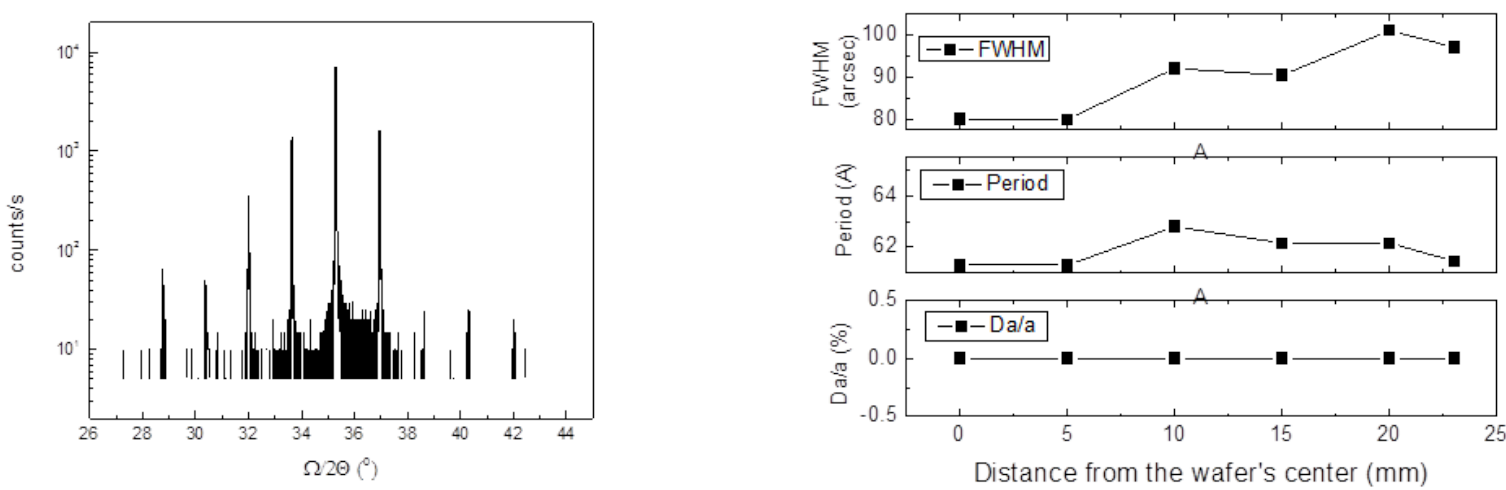

Figure 5. HRXRD spectrum (left) and Lattice mismatch, period thickness, and FWHM of the 1st order T2SL peak (right) of the grown detector structure.

The structural properties of the grown T2SL material were evaluated by high-resolution X-ray diffraction (HRXRD) measurements performed with a Philips double-crystal X-ray diffractometer using the $\mathrm{Cu}-\mathrm{K} \alpha 1$ line, which is shown in Figure 5. At the center of the 2" wafer, the overall periodicity of the T2SL absorbers was $61.3 \AA$. The width (full width at half maximum, FWHM) of the 1st satellite T2SL peak is 80 arcsec. There is no lattice mismatch between the GaSb substrate and the T2SL. To investigate the uniformity of the growth across the 2 " substrate, we performed HRXRD measurements across the wafers radius with 5 mm increments. The period thickness and average lattice constant of the T2SL varied insignificantly, implying high uniformity of the T2SL structure. The FWHM of the 1st order T2SL peak slightly increased towards the edge of the wafer suggesting the presence of a small temperature gradient across the wafer during growth.

It should be noted that the FWHM of the 1st T2SL satellite peak ( 80 arcsec) of the grown detector structure is larger than the FWHMs values typically cited for T2SL LWIR structures (30-40 arcsec). We attribute this to the p-type (Be) doping the of T2SL layers. Be-doping can broaden and even split the T2SL XRD peaks, as observed by Sankowska et al. ${ }^{28}$ Beryllium atoms change the strain in the pseudomorphically grown T2SL due to an average lattice constant change.

To verify the device cut-off wavelength, single element detectors were fabricated. Device fabrication was initiated with standard optical photolithograpy to define $410 \times 410 \mu \mathrm{m}$ square mesa devices with apertures ranging from 25 to $300 \mu \mathrm{m}$. The mesas were etched by IPC with a $\mathrm{BCl}_{3}$ - based chemistry to an etch depth of $\sim 9 \mu \mathrm{m}$, which corresponds to the middle of the bottom contact layer. Next, ohmic contacts were evaporated on the bottom and top contact layers using $\mathrm{Ti}(500 \AA) / \mathrm{Pt}(500 \AA) / \mathrm{Au}(3000 \AA)$ in both cases.

Finally, the devices were passivated by SU-8 2002 photoresist after a short (40 seconds) dip in a phosphoric 


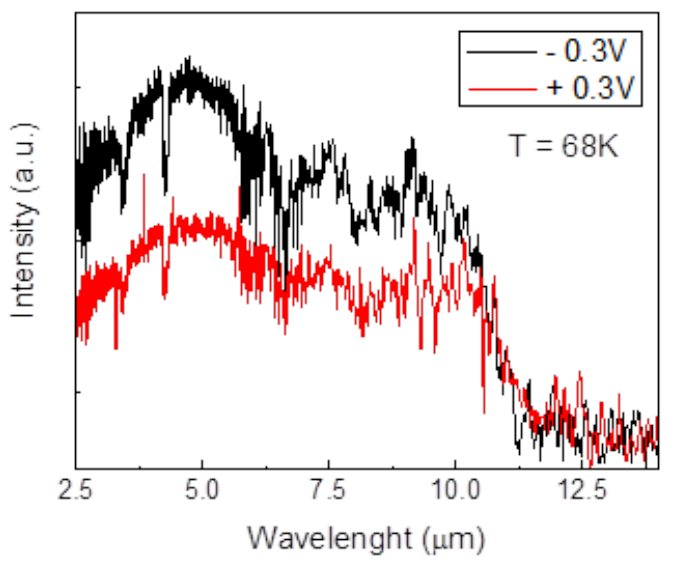

Figure 6. Spectral response (a.u.) measured at $68 \mathrm{~K}$ under negative and positive bias polarities from the T2SL detector structure. Both absorbers have the same thickness $(4 \mu \mathrm{m})$ and T2SL composition.

acid based solution $\left(\mathrm{H}_{3} \mathrm{PO}_{4}: \mathrm{H}_{2} \mathrm{O}_{2}: \mathrm{H}_{2} \mathrm{O}=1: 2: 20\right)$ intended to remove the native oxide film formed on the etched mesa sidewalls.

After fabrication, the devices were diced and mounted on 68 pin leadless chip carriers (LCC) for further characterization. Spectral response measurements were performed at $68 \mathrm{~K}$ with a Fourier transform IR spectrometer (FTIR) equipped with a glow-bar black body source. The detector spectral response curves, shown in Figure 6, were similar for both negative and positive bias polarities, as expected from the device design. The measured $50 \%$ cut-off wavelengths were $\sim 10.7 \mu \mathrm{m}$ and $\sim 10.4 \mu \mathrm{m}$ for the negative and positive applied biases, respectively (at $68 \mathrm{~K}$ ).

To provide feedback on the morphology and chemical composition of the etched and passivated sidewalls of the LWIR T2SL detectors with smaller features, $24 \mu \mathrm{m} \times 24 \mu \mathrm{m}$ mesas of $320 \times 256$ FPA were delineated from the same T2SL material with $\mathrm{BCl}_{3}$-based ICP.

\section{RESULTS AND DISCUSSION}

\subsection{Raman Spectroscopy of FPA Sidewalls}

Micro-Raman spectra of the $24 \mu \mathrm{m} \times 24 \mu \mathrm{m}$ mesa of T2SL FPA with $6.7 \mu \mathrm{m}$ etch depth are shown in Figure 7 . The spectra were measured at different points of the sample: the center of the mesa (1), two points on the sidewall $(2,3)$, the center of the etched trench $(4)$, and the crossing of the trenches (5). Spectrum 1 corresponds to the unetched SL, the strongest features are associated with the LO phonons of InAs at $\sim 237 \mathrm{~cm}^{-1}$. This is in agreement with the Raman selection rules: in crystals of zinc blende structure only LO phonons are allowed in backscattering from the (001) surface. ${ }^{4}$ GaSb LO phonons are weaker and broader due to the smaller thickness of GaSb layers in the SLs. (The SL thickness is much larger than the penetration depth of the laser light, therefore the substrate signal is suppressed in the spectra from the unetched region.) In contrast, spectrum 5 (from the deepest etched area) is dominated by the LO phonons of GaSb, associated with the GaSb substrate. InAs phonons are still present at lower intensity, which is probably due to the contribution of nearby sidewall regions. The spectra from the sidewall regions (2-4) show equally strong features of InAs LO and TO phonons. This is due to the deviation from backscattering geometry along the (001) direction. GaSb TO phonon features are also present, their intensity increases as the laser spot moves closer to the center of the etched trench (spectrum 4). This behavior can be explained by the increased contribution of the GaSb substrate or buffer layer in the deeper etched regions. Peaks associated with InAs phonons in the sidewall spectra are significantly broader compared to the unetched pixel top. This is due to structural disorder (poorer crystalline quality) on the sidewalls resulting from etching damage. 


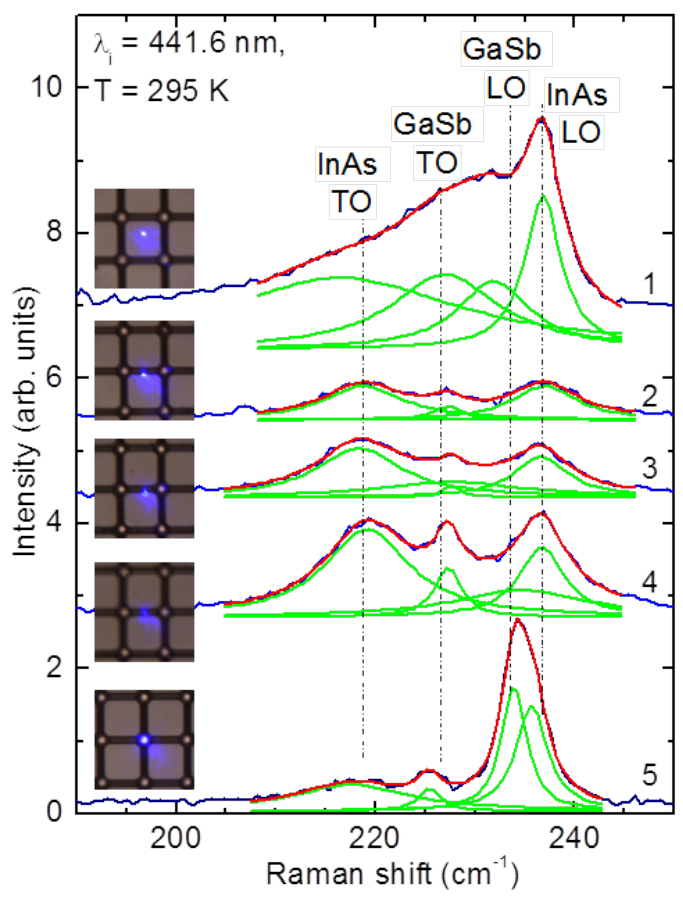

Figure 7. Micro-Raman spectra of a T2SL-FPA structure measured at different points. Lorentzian fitting was performed to distinguish different phonon features in the spectra (red lines - fitting results; green lines - contributions from individual phonon modes). The inset images show the location of the laser spot corresponding to the measured spectra. Dashed lines are guides to the eye.

It is important to point out here that the Raman data presented in Figure 7 is obtained under normal laser excitation in contrast to Fig. 1, where laser excitation was applied at $45^{\circ}$ with respect to the sample surface in order to reduce elastically scattered laser light collected by the objective. With the laser excitation at $45^{\circ}$, the polarizations of incident and scattered light can be independently controlled using polarization rotators and filters to effectively measure Raman scattering on transverse optical phonons (TO, corresponding to atoms oscillating perpendicular to phonon propagation) and longitudinal phonons (LO, corresponding to oscillations along phonon propagation), according to the Raman selection rules. Analysis of the Raman spectra from Fig. 1 demonstrated that TO phonon peaks can be utilized for characterization of the pixel sidewall quality. Raman peaks corresponding to both InAs and GaSb phonons carry information about material quality on the pixel sidewalls. This information is contained in both peak intensity and width. We can see that the peak width (FWHM) is increased (by $1.24 \mathrm{~cm}^{-1}$ and $1.68 \mathrm{~cm}^{-1}$ for InAs and GaSb phonons, respectively) at the sidewalls compared to the pixel top, likely due to defects introduced by the plasma etching process. Since both peak magnitude and width are affected, the phonon intensity is characterized by the area under the Raman peak (integrated intensity). The integrated intensity of the InAs peak is observed to decrease by $42 \%$ for InAs and $30 \%$ for GaSb on sidewalls, compared to the pixel top. This might suggest that InAs is more severely affected by the plasma etch than GaSb.

\subsection{AFM Characterization of T2SL FPA Sidewalls using Actoprobe High Aspect Ratio Probes}

Imaging a complete height profile of a $\sim 10 \mu \mathrm{m}$ deep trench requires an AFM scanner with extended vertical range. For AFM characterization of the FPA samples, Actoprobe used a scanner with $40 \mu \mathrm{m}$ vertical range. Figure 8 shows an T2SL test-sample (from Night Vision Inc., Pasadena, CA) with the AFM probe cantilever as seen through an optical microscope along with the measured height profiles. These were obtained using a high-aspect-ratio probe with a $\sim 10 \mu \mathrm{m}$ long tip, that was fabricated by Actoprobe (see Figure 3 ). The height profiles show a $\sim 9.2 \mu \mathrm{m}$ deep trench that is $10-13 \mu \mathrm{m}$ wide. The $28 \mu \mathrm{m}$ scan height profile shows the entire 

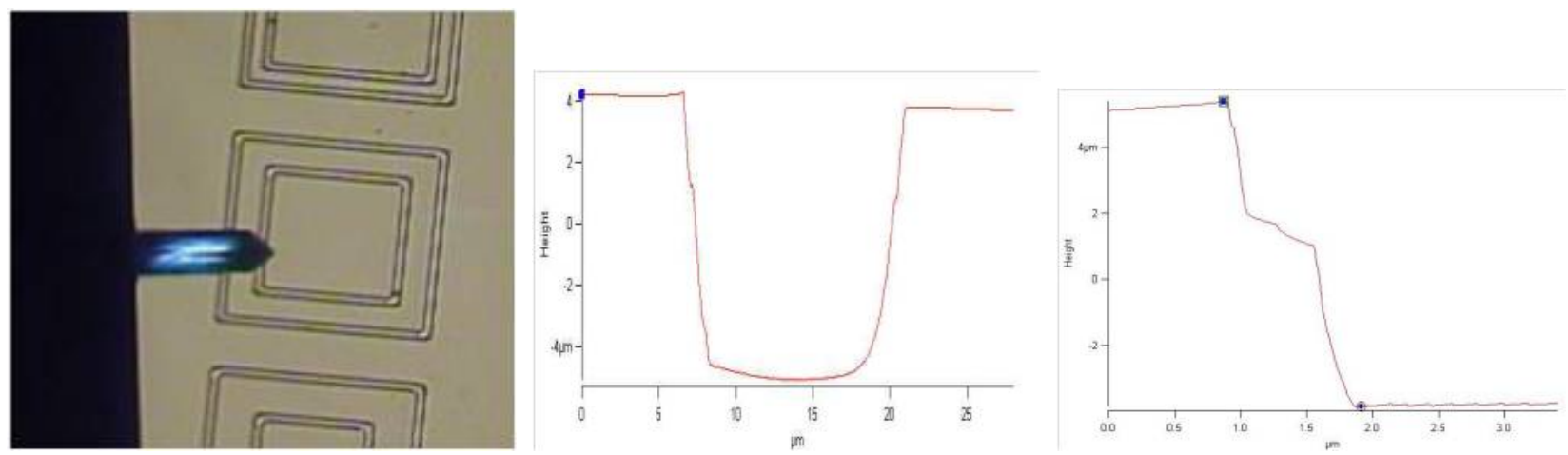

Figure 8. The T2SL test sample (provided by NVESD) with the AFM probe cantilever as seen through an optical microscope (left). Height profile of this T2SL test sample (from the topography scan in Figure 9). The $28 \mu \mathrm{m}$ scan (center) shows the entire trench. Height profile of a $5 \mu \mathrm{m}$ scan that shows the left sidewall in higher resolution and detail (right).
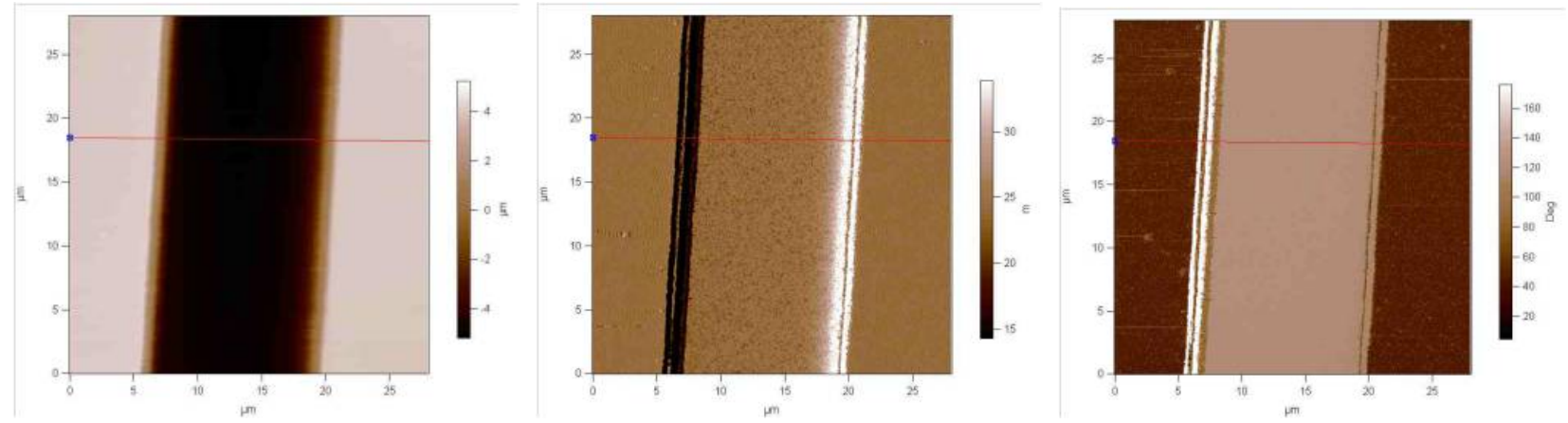

Figure 9. T2SL test sample (provided by NVESD): $28 \mu \mathrm{m}$ AFM scans over the entire trench using a scanner with extended vertical range $(40 \mu \mathrm{m})$ and a high-aspect-ratio probe tip with $\sim 10 \mu \mathrm{m}$ length fabricated by Actoprobe. Topography scan (left), amplitude (center) and phase map (right).

trench. There is a step visible at the left sidewall. A $5 \mu \mathrm{m}$ scan height profile shows the left sidewall in higher resolution and confirms the presence of the step. Figure 9 shows a $28 \mu \mathrm{m}$ AFM scan of this T2SL test sample (topography, amplitude and phase).

In the $28 \mu \mathrm{m}$ height profile in Figure 8 (center), there is a difference between the left and right sidewall. The difference may be actually present in the sample or it may be an artifact of the AFM scan. The latter may be due to the small angle between the probe tip and the sidewall, which results in a true image of only one sidewall and a slightly distorted one of the other. Whether the difference is present in the sample or is due to an AFM artifact can be determined from AFM scans with the sample rotated by $180^{\circ}$. If the shape differences of the sidewalls follow the sample rotation, then the sidewalls are actually different, whereas if the shapes switch from one to the other sidewall, then they are due to an AFM artifact.

Figure 10 shows a more detailed analysis of the left sidewall in a $5 \mu \mathrm{m}$ scan and a $2.5 \mu \mathrm{m}$ scan as well as a $3 \mathrm{D}$ representation. Figure 11 shows an amplitude and phase scan associated with the $2.5 \mu \mathrm{m}$ scan, that reveal more details of the step in the left sidewall.

\section{SUMMARY AND CONCLUSION}

In conclusion, we characterized the sidewalls of $24 \mu \mathrm{m} \times 24 \mu \mathrm{m}$ T2SL FPA structures by AFM, confocal microscopy and Raman spectroscopy. The measurements provide information about chemical and morphological characteristics of the FPA pixel sidewalls. Substantial differences in the Raman spectra were observed on T2SL FPA pixel sidewalls compared to the pixel center, which allowed a quantitative characterization of the crystal structure's degradation due to etch damage with respect to their constituents (InAs and GaSb). Large morphological changes were detected using AFM at different depths (from 0 up to $9.2 \mu \mathrm{m}$ ) on the pixel sidewalls in 

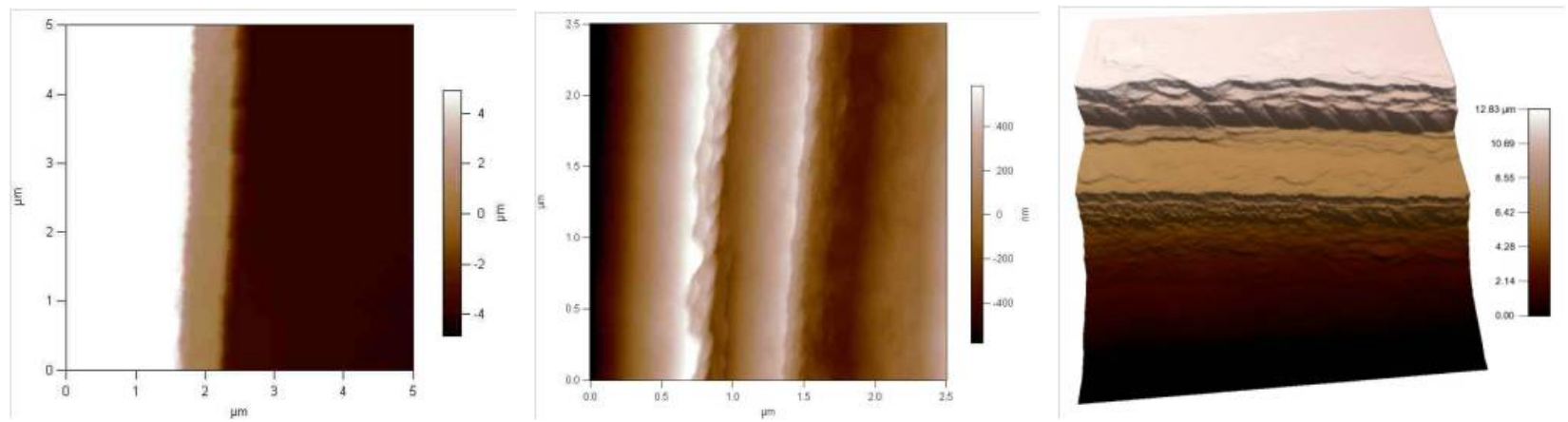

Figure 10. T2SL test sample (provided by NVESD): Higher resolution scans of the left sidewall. The $5 \mu \mathrm{m}$ topography scan (left) shows the step at $\sim 3.4 \mu \mathrm{m}$ below the top, as shown also in the height profiles in Figure 8 . The $2.5 \mu \mathrm{m}$ scan (center) shows the step in more detail, but the height scale is tilted due to attenuation. 3D image of the left sidewall with a correct height scale (right). It also shows the step observed in Figure 8.
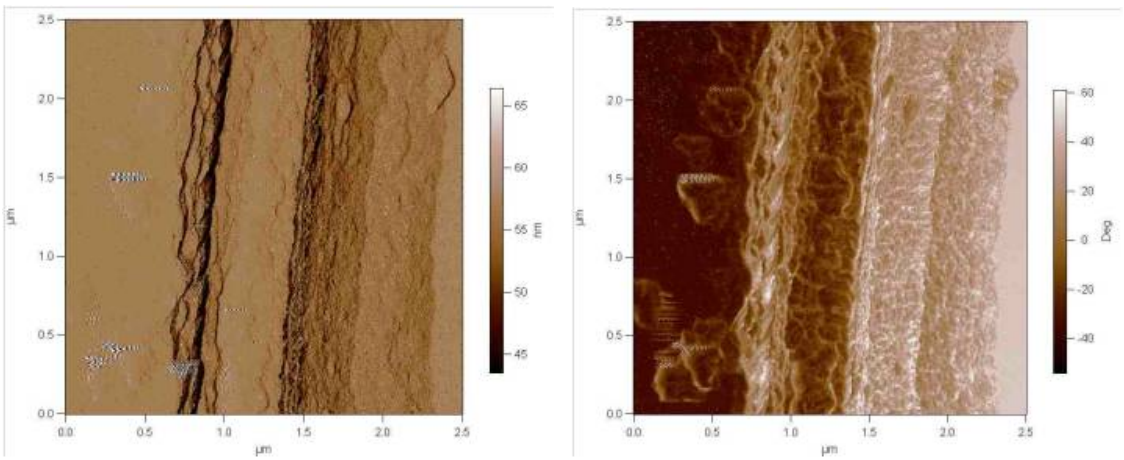

Figure 11. T2SL test sample (provided by NVESD): Higher resolution scans of the left sidewall. Amplitude (left) and Phase map (right) associated with the $2.5 \mu \mathrm{m}$ scan in Figure 10 (center). These images show more details of the step in the left sidewall.

topography, amplitude and phase. Our team found that AFM and optical tip-enhanced imaging, even though not fast, are very useful for detailed FPA studies in small regions of interest.

We can conclude that Raman spectroscopy is a useful tool to characterize the pixel sidewalls. Further, we plan to improve the signal-to-noise ratio and spatial resolution of the system. The application of a Krypton Ion Laser (instead of He-Cd) will increase the interaction volume for Raman scattering because of its longer wavelength (647.1 nm and $752.5 \mathrm{~nm}$ compared to $441.6 \mathrm{~nm}$ ). A single grating Raman spectrometer (e.g. Renishaw) will improve the instrument throughput. An objective with higher NA (0.85-0.95) will increase the Raman signal collection and spatial resolution. One of our main goals is to simplify optical imaging and Raman spectroscopy in the nano-scale (single molecular scale) and make it affordable and user-friendly to AFM customers. Actoprobes $\mathrm{R} \& \mathrm{D}$ is focussing on the development of a special AFM probe, AFM Active Optical Probe, AAOP, which combines a diode laser and photodetector with an AFM probe as a monolithic device. ${ }^{2,3,20}$ The device integrates a tunable Raman laser and a tunable Raman detector on an AFM cantilever. It will dramatically improve the data quality for Tip Enhanced Raman spectroscopy (TERS) and microscopy. The AAOP will facilitate TERS and Raman imaging with nanometer resolution with as little complexity as conventional AFM imaging. Our team considers Quantum $\operatorname{dot}^{35}$ and Quantum Dash ${ }^{27}$ based laser materials to be good candidates for this optoelectronic device because of their unique optoelectronic properties. In particular, the broad gain spectrum of $\mathrm{QD}^{35}$ and QDash $^{27}$ lasers allows tuning over $300 \mathrm{~nm}$ spectral range and fabricating diode lasers with peak gain between $1 \mu \mathrm{m}$ and $2 \mu \mathrm{m} .{ }^{1,27}$ The small carrier induced refractive index change ${ }^{33,34}$ and low jitter ${ }^{12,31}$ are important for pulse applications of AAOP. 


\section{ACKNOWLEDGEMENT}

This work was performed, in part, at the Center for Integrated Nanotechnologies, an Office of Science User Facility operated for the U.S. Department of Energy (DOE) Office of Science by Los Alamos National Laboratory (Contract DE-AC52-06NA25396) and Sandia National Laboratories (Contract DE-AC04-94AL85000). The authors would like to thank the faculty and technical staff of the Center for High Technology Materials (CHTM) at the University of New Mexico for fruitful discussion and technical support. Approved for Public Release 15-MDA-8215 (22 April 15).

\section{REFERENCES}

[1] Ganesh Balakrishnan, Shenghong Huang, Thomas J Rotter, Andreas Stintz, LR Dawson, Kevin J Malloy, $\mathrm{H} \mathrm{Xu}$, and DL Huffaker. $2.0 \mu \mathrm{m}$ wavelength inas quantum dashes grown on a gaas substrate using a metamorphic buffer layer. Applied physics letters, 84(12):2058-2060, 2004.

[2] T. Busani, T. J. Rotter, P. Rathi, F. Jaeckel, and Alexander A. Ukhanov. Army sbir phase i final report. 2014.

[3] T. Busani, T. J. Rotter, P. Rathi, F. Jaeckel, and Alexander A. Ukhanov. Army sbir phase i option final report. 2015.

[4] M. Cardona and G. Guentherodt. Light Scattering in Solids II. Springer, Heidelberg, 1982.

[5] P-Y Delaunay and Manijeh Razeghi. Spatial noise and correctability of type-ii inas/gasb focal plane arrays. Quantum Electronics, IEEE Journal of, 46(4):584-588, 2010.

[6] N Gautam, M Naydenkov, S Myers, AV Barve, E Plis, T Rotter, LR Dawson, and S Krishna. Three color infrared detector using inas/gasb superlattices with unipolar barriers. Applied Physics Letters, 98(12):121106, 2011.

[7] Nutan Gautam, Stephen Myers, Ajit V Barve, Brianna Klein, Edward P Smith, Dave R Rhiger, Ha Sul Kim, Zhao-Bing Tian, and Sanjay Krishna. Barrier engineered infrared photodetectors based on type-ii inas/gasb strained layer superlattices. Quantum Electronics, IEEE Journal of, 49(2):211-217, 2013.

[8] CH Grein, ME Flatte, JT Olesberg, SA Anson, L Zhang, and TF Boggess. Auger recombination in narrowgap semiconductor superlattices incorporating antimony. Journal of applied physics, 92(12):7311-7316, 2002.

[9] SD Gunapala, DZ Ting, CJ Hill, Jean Nguyen, Alex Soibel, SB Rafol, SA Keo, JM Mumolo, MC Lee, JK Liu, et al. Demonstration of a 10241024 pixel inas-gasb superlattice focal plane array. Photonics Technology Letters, IEEE, 22(24):1856-1858, 2010.

[10] A Haddadi, SR Darvish, G Chen, AM Hoang, BM Nguyen, and M Razeghi. High operability 1024 â 1024 long wavelength type-ii superlattice focal plane array. IEEE J. Quant. Electron, 48:221-228, 2012.

[11] AM Hoang, G Chen, A Haddadi, and M Razeghi. Demonstration of high performance bias-selectable dualband short-/mid-wavelength infrared photodetectors based on type-ii inas/gasb/alsb superlattices. Applied Physics Letters, 102(1):011108, 2013.

[12] H.Su. Ph.d. dissertation. 2004.

[13] Edward Kwei-wei Huang, Minh-Anh Hoang, Guanxi Chen, Shaban Ramezani-Darvish, Abbas Haddadi, and Manijeh Razeghi. Highly selective two-color mid-wave and long-wave infrared detector hybrid based on type-ii superlattices. Optics letters, 37(22):4744-4746, 2012.

[14] Edward Kwei-wei Huang and Manijeh Razeghi. World's first demonstration of type-ii superlattice dual band 640x512 lwir focal plane array. In SPIE OPTO, pages 82680Z-82680Z. International Society for Optics and Photonics, 2012.

[15] Jianliang Huang, Wenquan Ma, Yulian Cao, Yang Wei, Yanhua Zhang, Kai Cui, Gongrong Deng, and Yanli Shi. Mid wavelength type ii inas/gasb superlattice photodetector using sioxny passivation. Japanese Journal of Applied Physics, 51(7R):074002, 2012.

[16] JH Jang, W Zhao, JW Bae, D Selvanathan, SL Rommel, I Adesida, A Lepore, M Kwakernaak, and JH Abeles. Direct measurement of nanoscale sidewall roughness of optical waveguides using an atomic force microscope. Applied physics letters, 83(20):4116-4118, 2003. 
[17] A Khoshakhlagh, JB Rodriguez, E Plis, GD Bishop, YD Sharma, HS Kim, LR Dawson, and S Krishna. Bias dependent dual band response from inas/ga (in) sb type ii strain layer superlattice detectors. Applied Physics Letters, 91(26):263504-263504, 2007.

[18] Donald F. King, Jason S. Graham, Adam M. Kennedy, Richard N. Mullins, Jeffrey C. McQuitty, William A. Radford, Thomas J. Kostrzewa, Elizabeth A. Patten, Thomas F. McEwan, James G. Vodicka, and John J. Wootan. 3rd-generation mw/lwir sensor engine for advanced tactical systems. Proc. SPIE 6940, Infrared Technology and Applications XXXIV, page 69402R, April 22, 2008.

[19] Jason Osborne, Shuiqing Hu, Haiming Wang, Yan Hu, Jian Shi, Sean Hand, and Chanmin Su. High-speed atomic force microscopy for patterned defect review. In SPIE Advanced Lithography, pages 86813C-86813C. International Society for Optics and Photonics, 2013.

[20] T. J. Rotter F. Jaeckel P. Rathi, T. Busani and Alexander A. Ukhanov. Nsf sbir phase i final report. 2015.

[21] E Plis, SS Krishna, EP Smith, S Johnson, and S Krishna. Voltage controllable dual-band response from inas/gasb strained layer superlattice detectors with nbn design. Electronics letters, 47(2):133-134, 2011.

[22] E Plis, JB Rodriguez, HS Kim, G Bishop, YD Sharma, LR Dawson, S Krishna, SJ Lee, CE Jones, and Vishnu Gopal. Type ii inas/gasb strain layer superlattice detectors with p-on-n polarity. Applied Physics Letters, 91(13):133512-133512, 2007.

[23] R. Rehm, M. Walther, J. Schmitz, J. Fleissner, S. Kopta, F. Fuchs, W. Cabanski, and J. Ziegler. J. Cryst. Growth, 278:150-161, 2005.

[24] Robert Rehm, Martin Walther, Frank Rutz, Johannes Schmitz, Andreas Wörl, Jan-Michael Masur, Ralf Scheibner, Joachim Wendler, and Johann Ziegler. Dual-color inas/gasb superlattice focal-plane array technology. Journal of electronic materials, 40(8):1738-1743, 2011.

[25] David R Rhiger. Performance comparison of long-wavelength infrared type ii superlattice devices with hgcdte. Journal of electronic materials, 40(8):1815-1822, 2011.

[26] A Rogalski. Rep. Prog. Phys., page 68, 2005.

[27] Thomas J. Rotter. Ph.d. dissertation. 2007.

[28] I Sankowska, A Jasik, J Kubacka-Traczyk, JZ Domagala, and K Regiński. Role of beryllium doping in strain changes in ii-type inas/gasb superlattice investigated by high resolution x-ray diffraction method. Applied Physics A, 108(2):491-496, 2012.

[29] DL Smith and C Mailhiot. Proposal for strained type ii superlattice infrared detectors. Journal of Applied Physics, 62(6):2545-2548, 1987.

[30] A. Soibel, S. D. Gunapala, S. V. Bandara, J. K. Liu, J. M. Mumolo, D. Z. Ting, C. J. Hill, and J. Nguyen. Large format multicolor qwip focal plane arrays. Proc. SPIE 7298, Infrared Technology and Applications $X X X V$, page 729806, May 07, 2009.

[31] Hui Su and Luke F Lester. Dynamic properties of quantum dot distributed feedback lasers: high speed, linewidth and chirp. Journal of Physics D: Applied Physics, 38(13):2112, 2005.

[32] David Z-Y Ting, Cory J Hill, Alexander Soibel, Sam A Keo, Jason M Mumolo, Jean Nguyen, and Sarath D Gunapala. A high-performance long wavelength superlattice complementary barrier infrared detector. $A p$ plied Physics Letters, 95(2):023508-023508, 2009.

[33] AA Ukhanov, A Stintz, PG Eliseev, and KJ Malloy. Comparison of the carrier induced refractive index, gain, and linewidth enhancement factor in quantum dot and quantum well lasers. Applied physics letters, 84(7):1058-1060, 2004.

[34] AA Ukhanov, RH Wang, TJ Rotter, A Stintz, LF Lester, PG Eliseev, and KJ Malloy. Orientation dependence of the optical properties in inas quantum-dash lasers on inp. Applied physics letters, 81(6):981-983, 2002.

[35] Alexander A. Ukhanov. Ph.d. dissertation. 2004.

[36] Masahiro Watanabe, Shuichi Baba, Toshihiko Nakata, Takafumi Morimoto, and Satoshi Sekino. A novel afm method for sidewall measurement of high-aspect ratio patterns. In SPIE Advanced Lithography, pages 69220J-69220J. International Society for Optics and Photonics, 2008.

[37] Yajun Wei, Aaron Gin, Manijeh Razeghi, and Gail J Brown. Type ii inas/gasb superlattice photovoltaic detectors with cutoff wavelength approaching $32 \mu \mathrm{m}$. Applied Physics Letters, 81(19):3675-3677, 2002. 
[38] Yajun Wei, Andrew Hood, Haiping Yau, Aaron Gin, Manijeh Razeghi, Meimei Z Tidrow, and Vaidya Nathan. Uncooled operation of type-ii inas/gasb superlattice photodiodes in the midwavelength infrared range. Applied Physics Letters, 86(23):233106-233106, 2005. 